\title{
Quasicrystal approximants with novel compositions and structures
}

\author{
M. Mihalkovič ${ }^{1}$ and M. Widom, \\ Department of Physics, Carnegie Mellon University, Pittsburgh, PA 15213 \\ ${ }^{1}$ also at: Institute of Physics, Slovak Academy of Sciences, 84228 Bratislava, Slovakia
}

\begin{abstract}
We identify several new quasicrystal approximants in alloy systems in which quasicrystals have not been previously reported. Some occur in alloys with large size contrast between the constituent elements, either containing small Boron atoms, or large $\mathrm{Ca} / \mathrm{Eu}$ atoms, leading to quasicrystal structures quite different from currently known systems where the size contrast is smaller. Another group of the approximants are layered Frank-Kasper structures, demonstrating competition between decagonal and dodecagonal ordering within this family of structures.
\end{abstract}

\section{INTRODUCTION}

Axial quasicrystals are structures that possess one axis of crystallographically forbidden rotational symmetry. The structure is quasiperiodic in the plane perpendicular to this axis, but can be periodic in the direction parallel to the axis. Since they never exactly repeat, their lattice constant can be thought of as infinite within the quasiperiodic plane. Along the periodic direction the lattice constant is finite and might be fairly small.

Quasicrystal approximants are ordinary, though complex, crystals whose local structural motifs naturally extend to quasiperiodic structures with the crystallographically forbidden rotational symmetries [1]. Approximants reproduce within their unit cell a portion of an aperiodic structure, certain special fragments of which can be extended periodically while maintaining reasonable local atomic structures. Generally approximants have large lattice parameters in the directions in which the corresponding quasicrystal is quasiperiodic.

Structures that possess at least one large lattice parameter are thus candidates for being a quasicrystal approximant. If, in addition they possess a short lattice parameter orthogonal to their large one(s) this direction is a candidate for being an axis of high rotational symmetry in an axial quasicrystal.

Experimentally observed axial quasicrystal symmetries are octagonal (8x), decagonal $(10 \mathrm{x})$ and dodecagonal $(12 \mathrm{x})$. To-date stable decagonal quasicrystals are known in the compounds AlCoNi, AlCoCu, AlMnPd, AlCrNi, AlNiRu, AlCuRh, AlFeNi, ZnMgDy, $\mathrm{AlCrFe}, \mathrm{GaFeCuSi}, \mathrm{AlCuCr}$ and possibly others. In all these cases, the structures are believed to be layered, with a basic $4 \AA$ unit consisting of two atomic layers that are either stacked periodically or with some modulation leading to periodicities of $4,8,12$ and $16 \AA$. Perpendicular to the axis the structures are quasiperiodic. Some observed metastable dodecagonal quasicrystals occur in Ta-Te, Ni-Cr, Bi-Mn, Ni-V.

In the process of searching for bulk metallic glass-forming compounds $[2,3]$ we developed a database of intermetallic structures which now contains about 1000 structures drawn mainly from standard references $[4,5,6,7]$. The data base contains the Pearson symbol of each structure (e.g. $\mathrm{B}_{4} \mathrm{Mg}_{2} \mathrm{Ru}_{5}$ has Pearson symbol oP22 indicating an orthorhombic Primitive cell with 22 atoms), the space group (e.g. number 55, or Pbam), as well as the lattice parameters and Wyckoff coordinates.

We screened this database for structures that contain one short lattice parameter of $5 \AA$ or less and one long lattice parameter of $8 \AA$ or more. Owing to the presence of at 

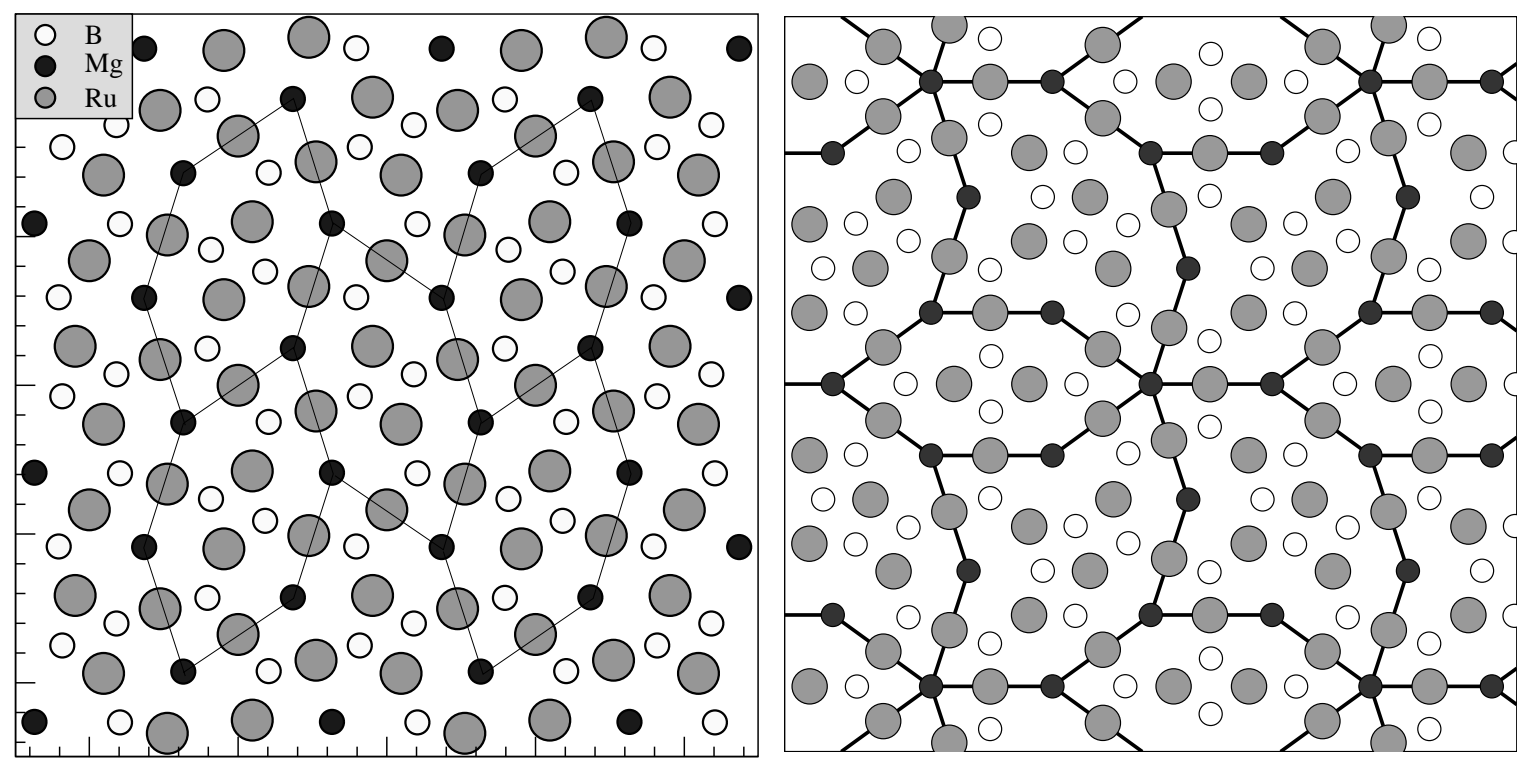

Figure 1: (a) $\mathrm{B}_{4} \mathrm{Mg}_{2} \mathrm{Ru}_{5}$ approximant. (b) Proposed model quasicrystal. Large and small circles indicate upper and lower layers. Black, gray and white indicate large, medium and small atoms, respectively.

least one long axis, these structures tend to have relatively large numbers of atoms per unit cell, as quoted in their Pearson symbols. We drew pictures of the structures projected along their short axis, then visually examined the picture. In many cases we observed the presence of local structural motifs of approximate 5-fold symmetry. We took special note of compounds that were chemically similar to the glass-forming compounds of interest (Al-Ca-Cu and B-Fe-Y-Zr).

When we found local 5 -fold symmetry we attempted to formulate a tile decoration model that would reproduce the observed structure and allow us to extend the structure to larger approximants or a complete quasicrystalline structure. We were able to do this in several cases.

Given a proposed quasicrystal model, we carried out total energy calculations using PAW potentials [8] in the generalized gradient approximation [9] as implemented by the program VASP $[10,11]$. Total energies of compounds of differing composition can be compared by measuring all energies relative to the tie-line connecting cohesive energies of pure elements. All structural energies were for fully relaxed structures and were converged to a precision of $1 \mathrm{meV}$ /atom. Because we relaxed the atomic volume, these energy differences are the structural enthalpy of formation, $\Delta H_{\text {for }}$, evaluated at temperature $\mathrm{T}=0 \mathrm{~K}$.

\section{BORON-CONTAINING PHASES}

One interesting case is $\mathrm{B}-\mathrm{Mg}-\mathrm{Ru}$ alloys, where $\mathrm{B}_{4} \mathrm{Mg}_{2} \mathrm{Ru}_{5}$.oP22 and $\mathrm{B}_{11} \mathrm{Mg}_{5} \mathrm{Ru}_{13} . \mathrm{OP} 62$ are two observed structures [12] that we believe are approximants of a $\mathrm{B}_{38} \mathrm{Mg}_{17} \mathrm{Ru}_{45}$ decagonal quasicrystal. Figure 1 shows the structure of oP22 and our proposed extension to a decagonal quasicrystal based on boat-hexagon tilings.

This compound is chemically unlike any presently known for quasicrystal formation. Notably, it contains a fairly large concentration of Boron. Owing to the prevalence of icosahedra in elemental Boron, researchers have long suggested that icosahedral quasicrystals might occur in Boron-rich compounds $[13,14,15,16,17,18]$, but none has 
yet been found.

Our proposed structure is promising because we have investigated the enthalpy of formation of other, larger hypothetical approximants. We consider how far these approximants lie from the convex hull of enthalpy versus composition for all known $\mathrm{B}-\mathrm{Mg}-\mathrm{Ru}$ structures, and find a large set of structures, nearly degenerate in energy, lying slightly above the convex hull. This is just the scenario envisioned for the entropic stabilization of quasicrystals, so we suggest that perhaps this compound will exhibit a thermodynamically stable high-temperature decagonal quasicrystal phase. We will describe the tiling energetics, including an evaluation of the energy of vertical structural degrees of freedom, breaking rigid 2-layer periodicity along the stacking (shortest periodic) axis. in an upcoming publication [19].

Likewise, $\mathrm{Al}_{8} \mathrm{CaCo}_{2} . \mathrm{OP} 44$ is an approximant of a hypothetical $\mathrm{Al}_{x} \mathrm{Ca}_{y} \mathrm{Co}_{z}$ decagonal phase. The structure here is quite similar to $\mathrm{B}-\mathrm{Mg}-\mathrm{Ru}$, with the substitutions $\mathrm{Mg} \rightarrow \mathrm{Ca}$, $\mathrm{B} \rightarrow \mathrm{Co}$ exactly reproducing the $\mathrm{B}-\mathrm{Mg}$ layer as a Co-Ca layer. However, the Al-content in the adjoining atomic layer is about $3 \times$ the $\mathrm{Ru}$ content of the adjoining layer in the B-based compound. Unlike the case of $\mathrm{B}-\mathrm{Mg}-\mathrm{Ru}$, where the star (S) tile is energetically unfavorable, leading to tilings of hexagons and boats only, for Al-Ca-Co the star tile is comparable in energy to the boat $(\mathrm{B})$ and hexagon $(\mathrm{H})$ tiles, leading to full Hexagon-Boat-Star tilings.

Due to the interest in finding genuinely B-rich quasicrystals, we screened our database to select high $\mathrm{B}$ concentration and found several candidate approximants. Noteworthy cases include $\mathrm{B}_{6} \mathrm{ReY}_{2}$.oP36, $\mathrm{B}_{7} \mathrm{ReY}_{3} . \mathrm{oC} 44$ and $\mathrm{B}_{4} \mathrm{CrY}$.oP24. All of these feature planar Boron pentagons, centered by a medium-sized transition metal. In the first two cases, a second pentagonal ring of Y-atoms surrounds the pentagonal ring of B-atoms.

We have not yet identified the proper manner to extend these structures to a full decagonal quasicrystal in a manner that achieves sufficiently low energy, but since they are all stable as BFeY alloys, and occur close-by in composition, we suspect there may be a unifying quasicrystal model to which they are approximants.

The most promising of these cases is $\mathrm{B}_{7} \mathrm{ReY}_{3}$, whose structure may be interpreted in terms of a pure tiling of skinny rhombuses, with B-Y pentagonal clusters centered by Re decorating tiling vertices, and alternating heights along short periodic direction along the tiling edges. Our best attempt extending this decoration to the fat tile is unstable by a moderate $20 \mathrm{meV}$ /atom energy. $\mathrm{B}_{2} \mathrm{C}_{2} \mathrm{Sc}$.oP20, another curious structure, is isostructural with $\mathrm{B}_{4}$ CrY.oP24 except that the pentagonal rings contain no medium-sized transition metal. In this compound, $\mathrm{C}$ and $\mathrm{B}$ share the pentagonal rings.

\section{RARE-EARTH-CONTAINING PHASES}

Another case that we consider promising for the occurence of an entropically stabilized decagonal quasicrystal is $\mathrm{Eu}-\mathrm{Cu}$ and the isostructural compound $\mathrm{Ca}-\mathrm{Cu}$. Here again we find two experimentally observed approximants, CuEu.oP8 and CuEu2.oP12 that allow us to devise decoration rules and extend the motif to a full decagonal quasicrystal, as shown in Fig. 2. Surprisingly, one of our larger approximant structures that we produced by hand, $\mathrm{Cu}_{4} \mathrm{Eu}_{9}$, turns out also to be predicted stable, though it has not been reported experimentally. The remaining approximants we tested lie slightly above the convex hull. In order for the quasicrystal to be stabilized entropically a mechanism introducing structural degrees of freedom along vertical direction is needed.

As in the case of the B-Mg-Ru system, this new alloy system is chemicaly unlike previously known quasicrystals, this time because it is rich in a Rare Earth element. An unique feature of this potential quasicrystal is striking simplicity of the decoration, with tiling edge length equal to the nearest neighbor interatomic distance. The small tile size is responsible for the large composition range of the family of approximants, spanning $x_{E u}=0.5 \rightarrow 1$ range of the Eu/Ca content (interestingly, both orthorhombic and monoclinic 

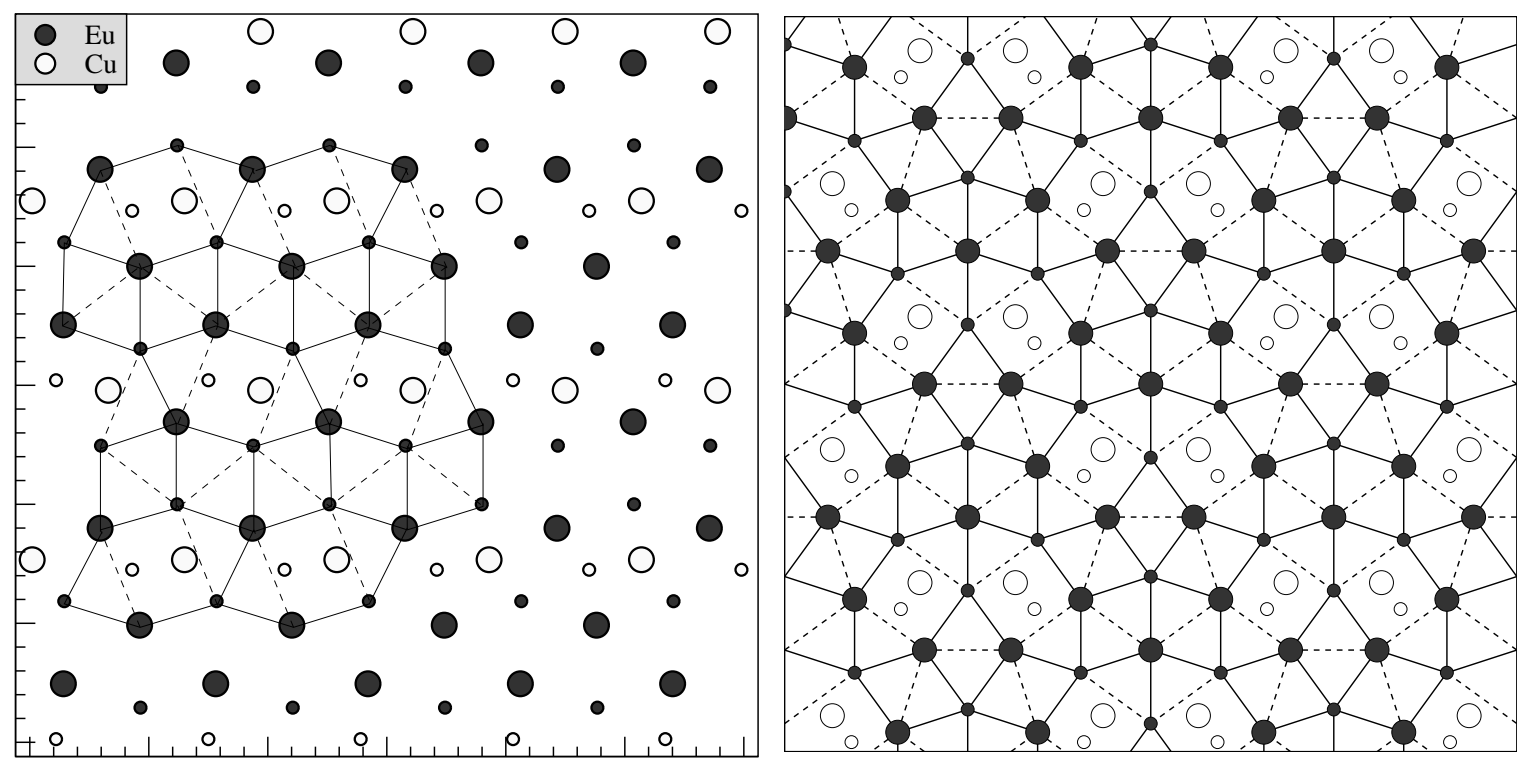

Figure 2: (a) $\mathrm{CuEu}_{2}$ approximant. (b) $\mathrm{Cu}_{4} \mathrm{Eu}_{9}$ approximant that we predict to be stable

$\mathrm{CaCu}$ structures, as well as $\mathrm{CuEu}$ with BFe structures, are valid approximants of the same quasicrystal family).

Unlike the Boron-based structures, where the basic tiles were drawn from HBS tilings, for $\mathrm{Cu}-\mathrm{Eu}$ the basic structure is based on a decoration of the rectangle-triangle (RT) tiling. The rectangle-triangle tiling is a $10 \times$ symmetric variant of the $12 \times$ symmetric square-triangle tiling which form dodecagonal quasicrystals. Interestingly, both these models have exact solutions for their entropy and phason elastic constants [20]. They also share the property that their phason degrees of freedom in general are quite complex involving non-local "zipper" updates [21].

\section{FRANK-KASPER STRUCTURES}

The final class of structures we mention is the Frank-Kasper family, characterized by tetrahedral close-packing. Constraints of close packing dictate that only special atomic environments with triangulated coordination shells occur, namely $Z=\{12,14,15,16\}$ with 12-16 nearest neighbors surrounding the central atom. Icosahedral quasicrystal

Frank-Kasper phases are represented by prototype $\mathrm{Al}_{6} \mathrm{Mg}_{11} \mathrm{Zn}_{11}$.cI162 phase [22], while the $\mathrm{Mg}_{4} \mathrm{Zn}_{7} \cdot \mathrm{mC110}$ phase is an approximant of a decagonal quasicrystal. The Laves phase of $\mathrm{MgZn}_{2} \cdot \mathrm{hP} 12$ may be viewed as a common approximant for both quasicrystal Frank-Kasper families.

The quasicrystal Frank-Kasper structures are pseudobinary, in that large atoms (e.g. $\mathrm{Mg})$ center highly-coordinated polyhedra $(Z=14,15$ or 16$)$ while smaller atoms (e.g. Al or $\mathrm{Zn}$ ) have icosahedral $Z=12$ coordination. In both icosahedral and decagonal structures about $60 \%$ of the atoms are of the smaller $Z=12$ coordinated type. The close relationship between decagonal and icosahedral FK structures was discussed by Roth and Henley [23].

We identified a family of layered (short axis $c \sim 5 \AA$ ) close-packed structures that interpolates between decagonal and dodecagonal ordering. In both cases, the structures may be viewed as simple decorations of square-triangle tiling (for the moment, we don't distiguish between isosceles/equilateral $\left(60^{\circ} / 72^{\circ}\right)$ triangles and between

squares/rectangles). Tiling vertices are occupied by pairs of atoms at $z= \pm 1 / 4$. Two kinds of tiling edges connect nearest-neighbor vertices: $e_{1}$ with an atom decorating the bond 

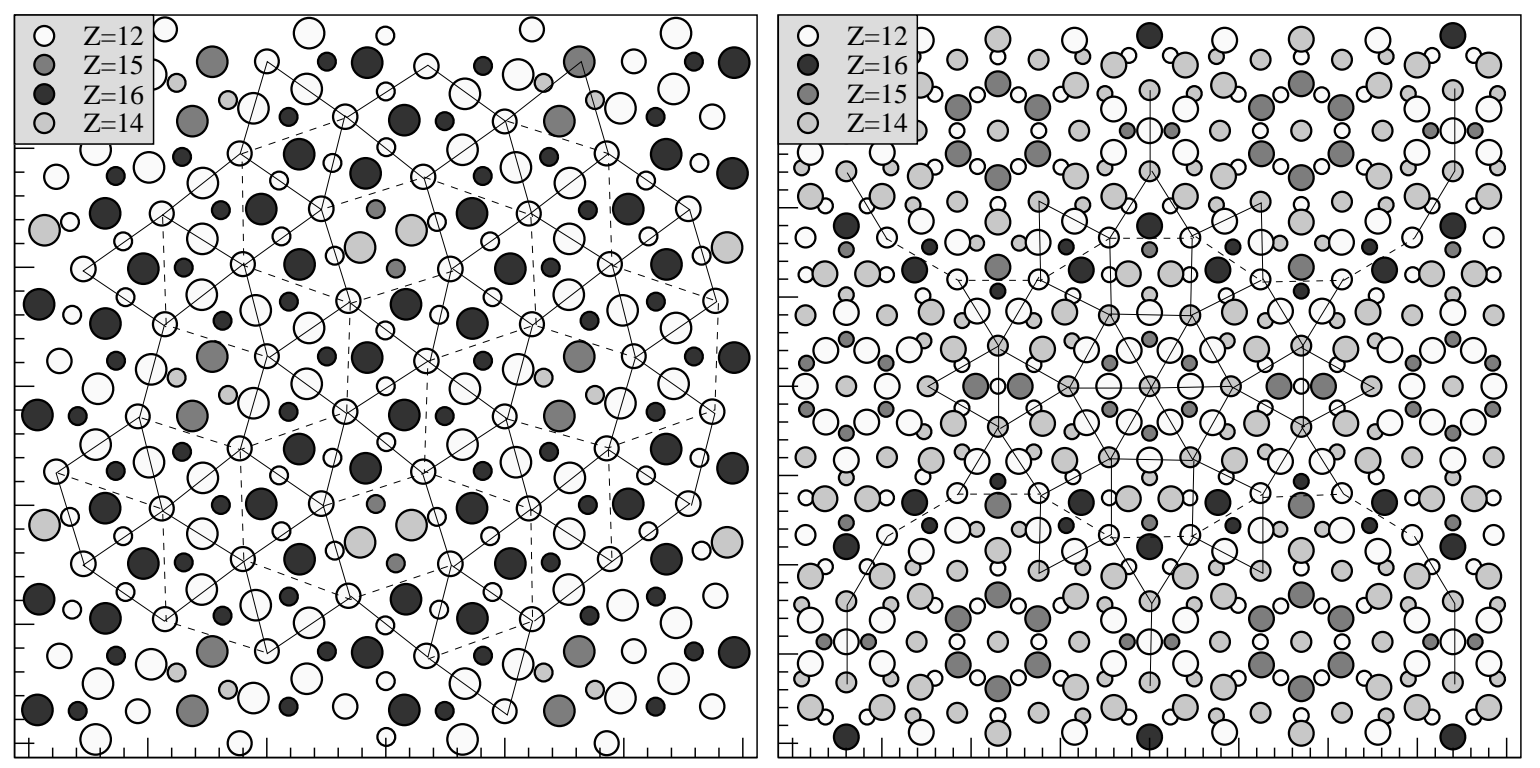

Figure 3: (a) Decagonal $\mathrm{Co}_{8} \mathrm{Mn}_{9} \mathrm{Si}_{3}$ and (b) dodecagonal $\mathrm{Mn}_{4} \mathrm{Si}$ structures. Tile edges: $e_{1}$ (solid) $e_{2}$ (dashed).

midpoint; $e_{2}$ containing no atom. Each triangle is decorated by one interior atom, each rectangle by four.

In the limit of pure dodecagonal order, all edges are of type $e_{1}$. Since all edges are equivalent, the triangles become equilateral and the rectangles are actually square. In the opposite limit of pure decagonal order, each triangle has two $e_{1}$ and one $e_{2}$ edge (so they are isosceles), and each rectangle has two $e_{1}$ and two $e_{2}$ edges. Squares possess a $4_{2}$ screw axis that rectangles lack. A packing rule requires adjacent tiles to share their common edge type.

Conversion of $e_{2}$ edges into $e_{1}$ by placing extra atoms on their midpoints converts $Z=12$ vertex polyhedra into $Z=14$. We define a parameter to measure the degree of dodecagonal $v s$. decagonal order in a layered FK approximant. First, identify the tile vertices by locating atom pairs at $z= \pm 1 / 4$ along the short axis. They must have coordination polyhedron $Z=12$ or $Z=14$. Then, define

$$
\zeta=N_{14} /\left(N_{12}+N_{14}\right)
$$

where $N_{Z}$ denote numbers of tiling vertices with $Z=12$ or $Z=14$ coordination. A structure is pure dodecagonal for $\zeta=1$, and pure decagonal for $\zeta=0$. Conversion of $e_{2} \rightarrow e_{1}$ produces $c$-height alternation conflicts for neighboring atoms inside the tiles, so it must be done non-locally.

Table I summarizes our results for several structures. Besides the $\zeta$ parameter, we provide numbers of triangles per unit cell $N_{t}$ and the $N_{t} / N_{s}$ ratio, where $N_{s}$ is number of "squares". The ideal $N_{t} / N_{s}$ ratios for decagonal and dodecagonal quasicrystal are $2(\sqrt{5}+1) \sim 6.47$ and $4 / \sqrt{3} \sim 2.31$ respectively. Structures with $\zeta>0$ have $N_{t} / N_{s}$ close to 2 , while the ratio is much larger than 2 for all but the smallest structures with $\zeta=0$.

Fig. 3 shows as examples a decagonal structure $\left(\mathrm{Co}_{8} \mathrm{Mn}_{9} \mathrm{Si}_{3} . \mathrm{OP} 74\right)$, and a dodecagonal $\left(\mathrm{Mn}_{4}\right.$ Si.oI186). The $e_{1}$ edges are shown as solid lines, $e_{2}$ dashed. While $\mathrm{Mn}_{4} \mathrm{Si}$ exhibits some icosahedra at the tiling vertices $(\zeta \sim 0.7)$, we regard it as a prominent dodecagonal approximant, due to the presence of large 12-fold clusters. These clusters actually extend beyond the dodecahedra outlined in the figure, so the clusters overlap and cover the structure. 
Table I: Dodecagonal and decagonal Frank-Kasper approximants

\begin{tabular}{l|c|c|c||l|c|c|c} 
structure & $N_{t}$ & $N_{t} / N_{s}$ & $\zeta$ & structure & $N_{t}$ & $N_{t} / N_{s}$ & $\zeta$ \\
\hline $\mathrm{Mg}_{4} \mathrm{Zn}_{7} . \mathrm{mC110}$ & 32 & 16 & 0 & $\mathrm{Co}_{17} \mathrm{Si}_{13} \nabla_{20} . \mathrm{mC50}$ & 12 & 6 & 0 \\
$\mathrm{Co}_{8} \mathrm{Mn}_{9} \mathrm{Si}_{3} . \mathrm{oP} 74$ & 20 & 10 & 0 & $\mathrm{Al}_{3} \mathrm{Nb}_{10} \mathrm{Ni}_{9} . \mathrm{OP} 52$ & 8 & 2 & 0 \\
$\mathrm{Cr}_{9} \mathrm{Mo}_{21} \mathrm{Ni}_{20} . \mathrm{OP} 56$ & 8 & 2 & 0.5 & $\mathrm{Mn}_{4} \mathrm{Si}_{\text {..oI186 }}$ & 14 & 2.333 & 0.692 \\
$\mathrm{CrFe} . \mathrm{tP} 30$ & 4 & 2 & 1 & & & &
\end{tabular}

Finally, note that the decagonal limit is optimal for pseudo-binary compounds with about $\sim 40 \%$ large and $60 \%$ small $(Z=12)$ atoms. In the compounds listed in Table I with $\zeta=0$, the elements $\mathrm{Mg}, \mathrm{Nb}, \mathrm{V}$ and $\mathrm{Mn}$ play role of the larger atoms, and occupy centers of $Z>12$ polyhedra. As $\zeta \rightarrow 1$, the fraction of $Z=12$ polyhedra decreases to about $1 / 3$, by converting $Z=12$ to $Z=14$. Meanwhile, volumes of the highly-coordinated polyhedra are only marginally bigger than those of the icosahedra, so that the dodecagonal structures are pseudo-monoatomic, by which we mean the atomic size contrast is small.

\section{ACKNOWLEDGEMENTS}

We wish to acknowledge useful discussions with C.L. Henley. This work was supported by NSF grant DMR-0111198 and benefited from metallic glass research funded by DARPA.

\section{REFERENCES}

1. V. Elser and C.L. Henley, Phys. Rev. Lett. 55, 2883-6 (1985).

2. M. Gao, G. Shifflet, M. Mihalkovič and M. Widom, unpublished, (2003).

3. M. Mihalkovič and M. Widom, in preparation, (2003).

4. P. Villars, A. Prince and H. Okamoto, Handbook of ternary alloy phase diagrams, ASM International, Materials Park, Ohio, (1995).

5. P. Villars, Pearson's Handbook, Desk Edition, ASM International, Materials Park, Ohio, (1997).

6. Binary Alloy Phase Diagrams, edited by T.B. Massalski, et al., ASM International, Materials Park, Ohio, (1990).

7. Desk Handbook: Phase Diagrams for Binary Alloys, edited by H. Okamoto, ASM International, Materials Park, Ohio, (2000).

8. G. Kresse and J. Joubert, Phys. Rev. B 59, 1758 (1999).

9. J.P. Perdew and Y. Wang, Phys. Rev. B 45, 13244 (1992).

10. G. Kresse and J. Hafner, Phys. Rev. B 47, RC558 (1993).

11. G. Kresse and J. Furthmuller, Phys. Rev. B 54, 11169 (1996).

12. K. Schweitzer and W. Jung, Z. Anorg. Allg. Chemie 530, 127-134 (1985).

13. P. Favio et al, Micros. Microan. Microstruct. 7, 225-34 (1996).

14. M. Takeda et al, $5^{\text {th }}$ International Conference on Quasicrystals, edited by edited by H. Okamoto, , World Scientific, Singapore, Materials Park, Ohio, (1995).

15. C. Weygand and J.-L. Verger-Gaugry, C. R. Acad Sci. II 320, 253-7 (1995).

16. K. Kimura, Mat. Sci. Eng. B 19, 67-71 (1993).

17. W.-J. Zhu and C. L. Henley, Europhys. Lett. 51, 133-9 (2000).

18. I. Boustani, A. Quandt and P. Kramer, Europhys. Lett. 36, 583-8 (1996).

19. M. Mihalkovič and M. Widom, Unpublished , (2003).

20. B. Nienhuis, Phys. Rep. 301, 271-92 (1998).

21. M. Oxborrow and M. Mihalkovič, Aperiodic '97, edited by M. de Boissieu, J.-L.

Verger-Gaugry and R. Currat, World Scientific, Materials Park, Ohio, (1997).

22. C. L. Henley and V. Elser, Phil. Mag. B 53, L59 (1986).

23. J. Roth and C. L. Henley, Phil. Mag. A 75, 861 (1997). 